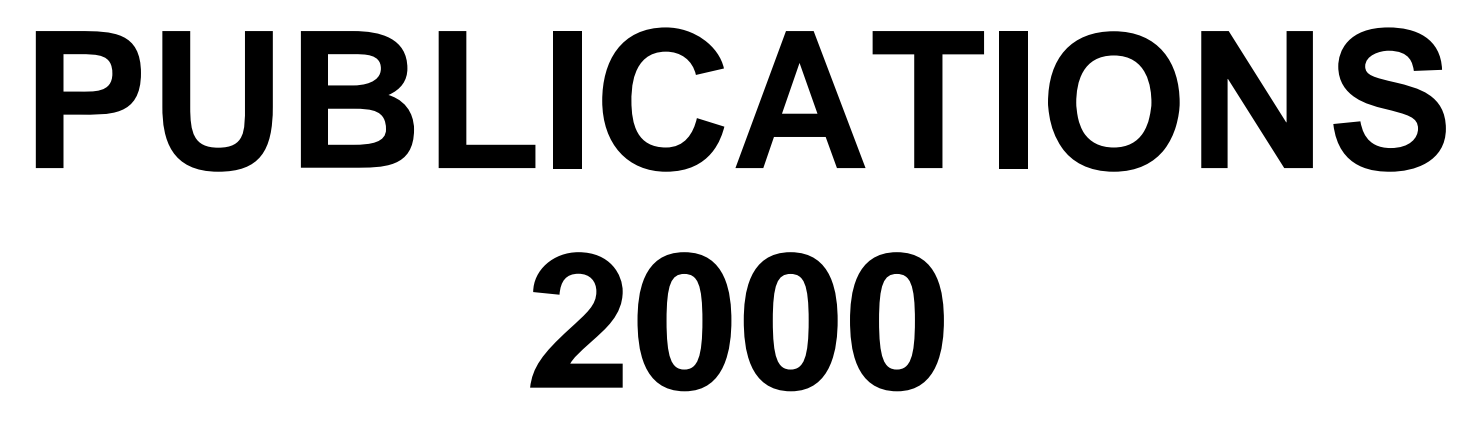




\title{
EFFECTS OF DIETARY RICE BRAN, LASALOCID, AND SEX OF CALF ON POSTPARTUM REPRODUCTION IN BRAHMAN COWS
}

\author{
S. M. Webb, A. W. Lewis, D. A. Neuendorff, and R. D. Randel
}

Background. Research with the postpartum cow shows that dietary fats and ionophore antibiotics may play key roles in improving postpartum reproductive efficiency of cattle. Dietary fat supplementation has been shown to increase weight gain, improve feed efficiency and stimulate follicular growth. Lasalocid, an ionophore antibiotic, increases the propionate:acetate ratio in the rumen which improves the metabolizable energy of concentrate diets; therefore, improving feed conversion and net energy for maintenance. The objective of this study was to evaluate the effects of ionophore supplementation in combination with fat supplementation during the postpartum period on reproductive endocrine function, follicular dynamics and postpartum reproductive efficiency. Sixty-eight mature Brahman cows with a body condition score of $6.2 \pm .7$ were randomly assigned to receive one of four nutritional treatments: 1) control, $9.2 \mathrm{lb}$ per head per day of $4: 1$ corn:soybean meal, 2) rice bran, $9.6 \mathrm{lb}$ per head per day corn:soybean meal:rice bran (5.2\% dietary fat), 3) lasalocid, $9.6 \mathrm{lb}$ per head per day of $4: 1 \mathrm{corn}$ :soybean meal $+200 \mathrm{mg}$ of lasalocid, 4) rice branlasalocid, $9.6 \mathrm{lb}$ per head per day corn:soybean meal:rice bran $(5.2 \%$ dietary fat $)+200 \mathrm{mg}$ of lasalocid. All animals were placed on Coastal bermudagrass (14\% CP) pastures overseeded with ryegrass $(24 \% \mathrm{CP})$ and were group-fed the experimental diets.

Research Findings. The addition of dietary lasalocid, rice bran, or their combination did not result in improvements in calf performance, cow body weight gain or cow body condition scores. Numbers of small, medium, and large follicles or the size of the largest follicle during the postpartum period were not influenced by dietary treatment. The interval from parturition to estrus had a tendency to be affected by dietary treatment. The number of days for return to estrus was lower for lasalocid $(41.72 \pm 3.71)$ and rice bran $(44.11 \pm 3.71)$ compared with rice bran-lasalocid $(51.56 \pm 3.82)$ and control $(53.67 \pm 3.71)$ groups. By d 60 after calving, the rice bran and lasalocid fed groups had a tendency for a higher percentage of cows returning to estrus compared to control or rice bran-lasalocid, and by d 70 after calving, estrus was observed in $100 \%$ of both rice bran and lasalocid groups. Cows fed lasalocid or rice bran were also more likely to have a functional corpus luteum by $\mathrm{d} 60$ as compared with the control or rice bran-lasalocid groups.

Application. Results of this study show that using rice bran or lasalocid in the postcalving diet did not significantly enhance follicular populations, but did decrease intervals to reproductively important endpoints. The combination of both lasalocid and rice bran lengthened the interval to 
reproductively important endpoints. 